\title{
Computed Tomography Imaging of the Coronary Arteries
}

\author{
G.J. Pelgrim, M. Oudkerk and R. Vliegenthart \\ Additional information is available at the end of the chapter \\ http://dx.doi.org/10.5772/54044
}

\section{Introduction}

In this chapter the possibilities of computed tomography (CT) for imaging of the coronary arteries are examined. Only in the last decades CT has entered the field of cardiac imaging, due to technical developments. First the history of CT in cardiac imaging is described. When did this technique enter clinical practice and what level of temporal and spatial resolution does it reach nowadays?

The goal of the CT technique described in this chapter is to image the coronary arteries. It is important to know how a CT scan for coronary imaging is made. This is discussed in the second part of the chapter. Contraindications to coronary CT angiography (CCTA) are put forward followed by the explanation of scan acquisition techniques. A detailed overview is provided regarding different CCTA scan protocols and types of acquisitions.

One of the main disadvantages of CCTA is the patient exposure to radiation to acquire the imaging data. Therefore, an important goal in CCTA imaging is to reduce the radiation dose while maintaining diagnostic image quality. There are multiple developments in the area of $\mathrm{CT}$ radiation reduction, which are discussed in the next section.

The diagnostic accuracy of CCTA has been investigated extensively in recent years. In this section the diagnostic accuracy of different CT scanner generations for calcium scoring and CCTA are expanded upon. This includes results for different parameters used in diagnostic accuracy studies, such as sensitivity and specificity.

What are the indications for CCTA examinations? This is the question which is answered in the fifth section. While this is a dynamic field, the main indications, supported by different consensus statements, are discussed. Approximately ten indications are described and ordered by relevance. Examples of different indications are shown by patient CCTA images. 
The future of cardiac CT is the last topic discussed. There are ample opportunities for future cardiac CT research such as CT perfusion imaging. These options are briefly mentioned.

\section{Computed tomography}

Computed tomography (CT) has been utilized in numerous fields in clinical practice since its invention in the 1970s. The first CT scanner was developed in 1971 by Geoffrey Hounsfield and installed at the Atkinson-Morley hospital in England. CT uses X-ray radiation to acquire 2D cross-sectional images of the body. X-ray imaging uses the different properties of different tissues to distinguish them in the image data. These images are acquired by a rapid 360 degree circular motion of the X-ray tube. The images are registered by the circular ray of X-ray detectors located in the gantry surrounding the patient. Then, a 2 dimensional reconstruction is made using the principle that an internal structure of the body can be made using multiple X-ray projections. To reconstruct a CT image, data from approximately $180^{\circ}$ of gantry rotation are required. From the start in 1972, CT has had an important role in diagnostics as non-invasive imaging technique.

In cardiac imaging, however, CT did not gain ground until developments in recent years. Early CT modalities were limited in their ability to display cardiac morphological information due to the interference of cardiac motion and spatial resolution. The diameter of coronary arteries varies from large, $3 \mathrm{~mm}$, to small, $1.5 \mathrm{~mm}$. Therefore, the spatial resolution of the angiography technique should be at least $1 \mathrm{~mm}$. [1] Temporal resolution was not sufficient to display the heart due to cardiac motion. Therefore, until recently, invasive coronary angiography (ICA) was the only accurate method for coronary imaging. [2, 3]

CT for cardiac imaging first entered the field with the development of electron beam computed tomography (EBCT) in the 1980s. EBCT was specifically developed for cardiac imaging, combining very high temporal resolution $(50-100 \mathrm{~ms})$ with prospective electrocardiographic (ECG) triggering. The high temporal resolution combined with ECG triggering greatly reduced cardiac motion artifacts. The main clinical application of EBCT was the quantification of coronary calcium deposits in a so-called calcium score. The calcium score is correlated with degree and severity of coronary artery disease (CAD) and is a strong predictor of coronary events. [4, 5] The application of calcium scoring is explained in more detail further in this chapter. Main limitation of EBCT is the spatial resolution of between $1.5 \mathrm{~mm}$ and $3 \mathrm{~mm}$ in the z-axis. This prevents EBCT to accurately determine the severity of CAD, especially in CCTA setting. After the introduction of multidetector CT (MDCT) scanners in 1990s (see below) and due to limited availability of EBCT scanners, EBCT was used less frequently and eventually replaced by MDCT systems from 2003 onwards.

The developments in CT were rapid, compared to other imaging fields in the last decades. These developments have led to considerably improved temporal and spatial resolution. MDCT scanners use multiple detectors to acquire the data, scanning multiple detector rows 
in one rotation. Already 4- and 16-row MDCT scanners caused a revolution in cardiac imaging, however diagnostic accuracy in terms of specificity was generally low. [6-10] Sensitivity and negative predictive value were already good. MDCT proved useful in evaluation of coronary anomalies and bypass graft patency. Although the 16-row MDCT scanners had improved spatial resolution, making detection and characterization of coronary plaques and coronary wall changes possible, high heart rates, stents and severely calcified arteries, however, affected the image quality negatively. [11, 12]

In 2004, the next generation of MDCT scanners was introduced, with 32, 40 and 64 slices, another step forward in speed of volume coverage. Compared to 16-row MDCT scanners, the gantry rotation time of 64-row MDCT scanners improved from $500 \mathrm{~ms}$ to $330 \mathrm{~ms}$. This translates in an improvement in temporal resolution from $250 \mathrm{~ms}$ to 165 $\mathrm{ms}$, as only half a rotation is needed to acquire the data required for the image reconstruction. The visualization of the coronary arteries was again markedly improved, with a high sensitivity and specificity achieved for evaluation of coronary stenosis. [13-15] Examinations in patients with high heart rates were reported to still yield diagnostic images, with the use of multisegment reconstruction algorithms, reducing influence of motion artifacts. [16, 17]

Dual source computed tomography (DSCT) is one of the latest improvements in CT imaging modalities. The DSCT scanners consist of 2 tube-detector systems mounted in the same gantry, off-set by 90 degrees (perpendicular). Compared with conventional single-source CT scanners, the temporal resolution of this CT scanner is twice as high. This is because the temporal resolution is equal to a quarter of the gantry rotation. The other parameters, such as gantry rotation time, are equal to single-source CT scanners. In DSCT, the temporal resolution is further improved to $83 \mathrm{~ms}$, further reducing the influence of motion artifacts on the image quality. Studies have shown an improvement in the assessment of the moving heart at a high heart rate without the need to use medication to control the heart rate during the examination. [18-22] Multiple studies have assessed the difference in image quality and accuracy of DSCT compared to 64-row scanners. [23-25] The higher temporal resolution resulted in better image quality and diagnostic accuracy.

Recent expansion of the detector width in MDCT has resulted in CT scanners with 256 and 320 detector rows. These systems allow for coverage of up to 320 slices during one rotation and in one heartbeat. This allows coverage of the whole heart in one gantry rotation. The principle of these CT scanners is the use of a cone beam. The X-ray tube can reach the detectors at the edges of the gantry readout, possibly displaying the whole heart in one scan. However, the 320-slice coverage comes at a cost as the temporal resolution is lowered to 350 $\mathrm{ms}$ and the edge of the scan range is prone to artifacts. [26-28]

The introduction of CT and different generations of CT scanners over time is described in Table 1. Continuously, new technologies are developed to improve the diagnostic performance of the CT technique for imaging the coronary arteries, including the spatial and temporal resolution. 


\begin{tabular}{|c|c|}
\hline \multicolumn{2}{|c|}{ Computed tomography development } \\
\hline Year & Technique \\
\hline 1971 & First computed tomography scanner \\
\hline $1980 \mathrm{~s}$ & Electron beam computed tomography (EBCT) \\
\hline $1990 \mathrm{~s}$ & Multidetector CT scanner \\
\hline 2004 & $32-, 40-$, 60-row multidetector CT \\
\hline 2006 & Dual source computed tomography (DSCT) \\
\hline 2007 & 256- and 320-slice CT \\
\hline
\end{tabular}

Table 1. Development in computed tomography with highlights through the years

\section{Imaging the coronary arteries by CCTA}

The goal of coronary CT angiography (CCTA) is to image the coronary arteries, detect coronary artery calcification, and evaluate coronary stenosis or occlusion. Final aim is to aid the cardiologist in determining the best patient treatment and management.

High quality images are the most important prerequisite in the diagnostic assessment of the coronary arteries. Certain factors need to be taken into account to ensure a high-quality CCTA examination in the correct patient. These factors include selecting the right patients for the examination, proper patient preparation, an adequate CT scanner, optimal CT scan protocol, including synchronization of the CT data with the ECG information and proper reconstruction of image data, and dedicated software for evaluation of the coronary CT images. Furthermore, a prerequisite for CCTA is the injection of iodinated contrast material to delineate the lumen of the coronary arteries. Therefore, an absolute contraindication for CCTA is an allergy for iodine. An overview of contraindications for CCTA are listed in table 2. [29] Apart from general contraindications for $C T$, there are some specific contraindications for CCTA, such as high or irregular heart rates.

\begin{tabular}{l}
\hline CCTA Contraindications \\
\hline Atrial fibrillation (permanent or at time of the study) \\
\hline Heart rate "/> 65 beats/minute refractory to heart-rate lowering agents \\
\hline Bigeminy, Trigeminy, high degree heart block \\
\hline Severe asthma \\
\hline Failed steroid preparation for contrast allergy \\
\hline Morbid obesity (body mass index "/>40) \\
\hline Palcium score "/> 1000 \\
\hline Inability to cooperate with scan acquisition and/or breath hold instructions
\end{tabular}

Table 2. Contraindications for coronary CTA 
As stated before, motion artifacts on CCTA are observed more frequently in patients with higher and irregular heart rates. This negatively affects the image quality and reliability of detecting or excluding coronary stenoses. For earlier generation 16- to 64-row MDCT scanners it has been proven that the highest image quality is achieved in patients with a low heart rate ( $<65$ beats per minute). [30-32] It was shown that breath hold at end-inspiration reduces the heart rate by (on average) 6 beats per minute, which can be tested prior to performing the CCTA acquisition. In case of a patient's heart rate higher than 70 per minute it is advised to reduce the heart rate by medication. This can be done by administration of intravenous injection of 5-25 mg metoprolol.

Patients are positioned on the CT table in supine position. The three ECG leads are attached to the patient body to acquire an adequate ECG tracing, which is synchronized with the raw image data. Furthermore, an 18-gauge intravenous-line is inserted to ensure a correct injection of the contrast agent. The actual acquisition protocol consists of three steps: a topogram, a determination of the contrast arrival time using a test contrast bolus or acquisition of repetitive images during contrast injection for bolus tracking and the actual CCTA scan.

First, a low-energy topogram is acquired to enable accurate positioning of the scan volume. Afterwards, a non-contrast scan can be performed to obtain a coronary artery calcification (CAC) score. The coronary calcium score is a calculation of the amount of coronary artery calcium. The most commonly used method for coronary calcium quantification is the calcium score according to Agatston. [33] A negative CT scan for coronary calcium shows no calcification in the coronaries. A positive test means CAD is present, also when a patient is asymptomatic. The amount of calcification, expressed in the calcium score, can help to predict the risk of coronary events. The extent of CAD is graded according to the calcium, shown in table 3 . The height of the calcium score is also strongly related to the risk of coronary heart disease. [34-37] At this moment, the strongest indication for coronary calcium scoring is in asymptomatic individuals at intermediate risk based on risk factors, to improve risk stratification. [38] For 64-row MDCT and earlier CT generations, a calcium score above 1000 is generally considered a contraindication for performing CCTA. The reason is twofold: patients with a very high calcium score have a considerable probability of having one or more significant stenosis, and severe calcifications cause blooming artifacts that limit the assessment of luminal narrowing.

Two techniques are available to correctly start the CCTA acquisition, based on arrival of contrast in the coronary arteries: the bolus tracking and the bolus timing technique. Bolus tracking involves a series of axial low-dose images to track the bolus of contrast material (every 2 seconds), monitoring the contrast enhancement in a region of interest (ROI) in the ascending aorta. The CCTA imaging sequence is initiated when the Hounsfield Unit (HU) in the ROI reaches a certain predefined level, usually $100 \mathrm{HU}$. The bolus timing technique involves an extra low-dose scan acquisition of a single slice. Here, a small contrast bolus followed by a saline flush is injected to determine the contrast arrival time. An axial low-dose image is generated every 2 seconds at a predefined ROI in the ascending aorta. The time between the start of the contrast injection and the arrival of contrast bolus in ROI is used as the scan delay for the actual CCTA. Both methods have similar results and have proven its usefulness in multiple research studies. 


\begin{tabular}{ll}
\hline Cardiac calcium score & Extent of CAD, risk of coronary events in the next $\mathbf{5}$ year \\
\hline 0 & No evidence of CAD, very low risk of coronary events \\
\hline $1-10$ & Minimal evidence of CAD, low risk of coronary events \\
\hline $11-100$ & Mild evidence of CAD, low-moderate risk of coronary events \\
\hline $101-400$ & Moderate evidence of CAD, moderate risk of coronary events \\
\hline$" />400$ & Severe evidence of CAD, high risk of coronary events \\
\hline
\end{tabular}

Table 3. Cardiac calcium score related to the extent of CAD

When the correct volume and scan delay have been selected, the actual CCTA scan can be performed. A volume dataset of the coronary arteries is required, covering the entire heart. The scan is acquired during breath hold. A contrast agent with a high concentration of iodine is used $(300 \mathrm{mg} / \mathrm{ml})$ to ensure adequate opacification of the coronary arteries. A total amount of $60-80 \mathrm{ml}$ of contrast agent is injected with an injection speed of approximately 4-6 $\mathrm{ml} / \mathrm{s}$, which is flushed by a saline bolus of $40-70 \mathrm{ml}(4-6 \mathrm{ml} / \mathrm{s})$.

The scanning parameters are different from vendor to vendor and per scanner generation. These parameters are beyond the scope of this chapter and can be obtained from the vendor of the CT scanner.

CCTA scans are usually acquired in spiral mode, with continuous acquisition of data throughout the whole cardiac cycle (see Figure 1). The quality of the reconstructed axial images is determined by multiple parameters.

The use of retrospective ECG-triggering enables the reconstruction of CCTA images at different time points in the R-R interval. The R-R interval is the time between two R-tops in a normal cardiac cycle. In previous studies it has been shown that the optimal visualization window for coronary imaging, nearly free of motion artifacts is mid-diastole, at $60 \%$ to $70 \%$ of the R-R interval. In patients with higher or irregular heart rates however, better image quality is usually obtained at $25 \%$ to $35 \%$ of the R-R interval.

Slice thickness is dependent on the parameters of the specific CT scanner. Thinner slices improve the quality of the 3-dimensional dataset and the quality of the reconstructed images; on the downside it also increases the image noise which could potentially limit the diagnostic accuracy of the CCTA examination.

The CCTA images are usually reconstructed with a medium smooth reconstruction kernel. The reconstruction kernel, also referred to as 'filter' or 'algorithm' by some CT vendors, is one of the most important parameters affecting the image quality. In general, there is a tradeoff between the spatial resolution and noise for each kernel. Smooth kernels generate images with low noise, resulting in lower spatial resolution. A sharp kernel however, generates images with high spatial resolution but also have increased noise levels. [39] Recently, iterative reconstruction techniques have been introduced. These techniques reduce image noise by iteratively comparing the acquired image to a modeled projection. This algorithm is 
developed to reduce the radiation dose and enhance tube current modulation. These reconstruction techniques have shown to reduce image noise and improve image quality. [40, 41]

Prospective ECG-triggering is a technique used in cardiac CT that uses forward-looking prediction of $\mathrm{R}$ wave timing (see Figure 1). This is step-and-shoot non-spiral acquisition without table motion during imaging. Main advantage of prospective ECG-triggering is the lower radiation dose compared to retrospective ECG-triggering, see below. A disadvantage is the possibility of non-diagnostic coronary artery segments in case of unexpected changes or irregularity in the heart rate, as retrospective manipulation of the CT image data is generally not possible. [42-44]

\section{Retrospective triggering}

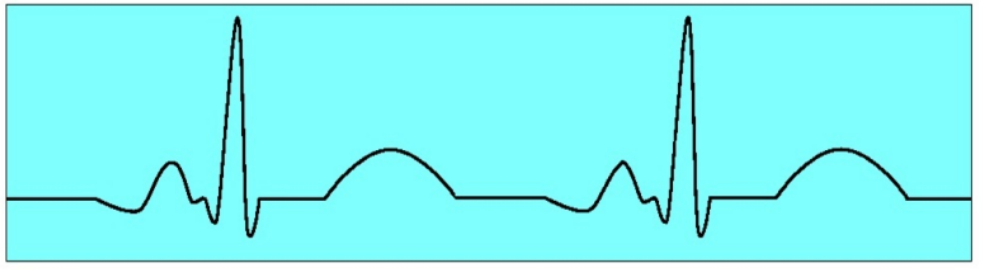

\section{Prospective triggering}

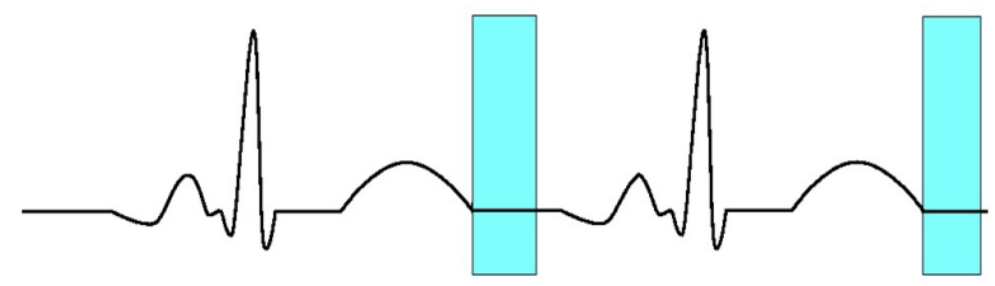

ECG gated tube modulation

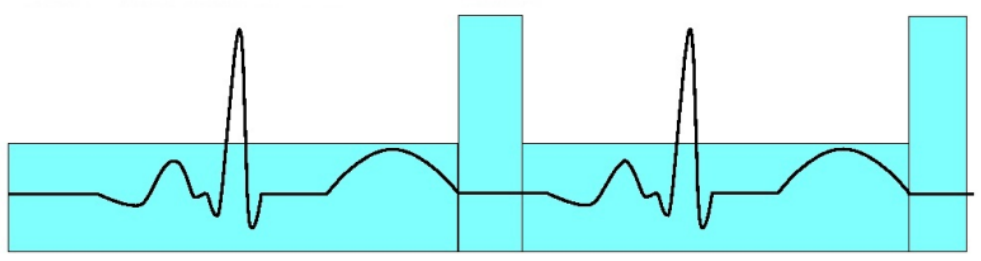

Figure 1. Different triggering techniques used to lower radiation dose. In retrospective triggering, the acquisition is constant and afterwards, the best cardiac phase is reconstructed for analysis. In prospective triggering, the acquisition is only performed during small parts of the cardiac phase, reducing radiation dose. In ECG-gated tube current modulation, the tube current is lowered during phases more likely to have motion artifacts and normal in the area of interest. 


\section{CCTA radiation exposure}

The retrospectively ECG-gated CT is associated with relatively high radiation dose because of low pitch and overlapping data. Effective doses that have been published vary between 2 and $30 \mathrm{mSv}$, a prospective triggering mode has an effective radiation dose of between 1-3 $\mathrm{mSv}$ in adults. [42-45]

Radiation is a potential threat, which can cause harm to the human body. Therefore, minimizing radiation exposure to patients is of critical importance for physicians. The International Commission on Radiological Protection (ICRP) estimated that chance of acquiring fatal radiogenic cancer in the adult population is approximately $0.005 \% / \mathrm{mSv}$. Furthermore, it is assumed that there is no safe amount of radiation; any radiation amount is potentially harmful. Thus, effort should be taken to keep radiation doses as low as possible while maintaining diagnostic scan relevance. This radiological principle is also expressed as 'As Low As Reasonably Achievable' or ALARA. Multiple factors influence the radiation exposure, including scanner type, tube current, tube voltage, ECG triggering, high pitch helical scanning, scan range, slice thickness, acquisition time, overlap and pitch. Those factors influencing the radiation exposure should all be taken into account, minimizing the radiation to the lowest level possible.

Tube current can be modified according to the patient body mass index (BMI). Higher tube current increases the amount of photons per exposure time, reducing the image noise, but at the same time increasing the radiation dose. Patients with higher BMI need higher tube current to reduce the noise level, generated by the higher amount of tissue penetrated. The tube current should only be increased to a level necessary for acquiring adequate diagnostic images.

Increasing tube voltage will lead to higher energy X-ray beams with higher tissue penetration, and substantially increased radiation dose. Generally, 100 to $120 \mathrm{KeV}$ tube voltages are sufficient for cardiac imaging. Only in really large patients, $140 \mathrm{KeV}$ could be used. Reducing tube voltage will reduce radiation dose in proportion to the square of changes in tube voltage. [46, 47]

ECG synchronization is of particular importance for the amount of radiation exposure. As aforementioned, in retrospective ECG triggering data are acquired throughout the whole cardiac cycle and only the data with the least motion are used for reconstruction. In prospective ECG triggering, the tube is only activated during a predefined cardiac phase with presumably the greatest likelihood of minimal cardiac motion. Because there is no radiation exposure during the remainder of the cardiac cycle, this results in a dose reduction potential of $31 \%-86 \%$. [25] In case of retrospective ECG triggering, ECG-based tube current modulation is an effective form of dose reduction (see Figure 1). This modulation utilizes the concept that coronary motion is least during end-systole and end-diastole. Therefore, the tube current is reduced by up to $96 \%$ during periods with presumably more cardiac motion and ramped up during diastole, when motion is at its lowest (for heart rates up to $65 \mathrm{bpm}$ ). [46] The image quality of phases with lower tube 
current is reduced, which is a downside of this technique. The potential dose reduction of ECG-gated tube current modulation is $13 \%-46 \%$. [25].

The introduction of the second generation DSCT scanners made high-pitch helical scanning possible. This prospectively ECG triggered technique involves a high speed of the patient table. Due to this high pitch, the heart can be scanned in a fraction of one heartbeat. This eliminates overlapping volume coverage of sequential sections. Early results show dose reductions of up to $80 \%$ and CCTA examinations with $<1 \mathrm{mSv}$ radiation dose. [25]

Scan range is a term that indicates the $\mathrm{z}$ range (length) of the body which is scanned. Therefore, the larger the scan range is set, the higher the radiation exposure will be. The scan range should be limited to the range that is ultimately necessary to clarify the diagnostic questionnaire. A scan of the coronaries should not include the aortic root unless this is specifically asked by the referring physician. This will limit unnecessary radiation exposure.

Some factors of minor importance are slice thickness, acquisition time, overlap and pitch. Thinner slices will increase radiation dose because of the larger overlap and lower pitch, which increases acquisition time. The thinner slices need equal amounts of data to have same contrast-to-noise ratio compared to thicker slices. Because of this effect, the table speed needs to be slower with more overlap. This results in higher radiation exposure. With wider detector ranges and dynamic scanning, these factors become less important. A summary of important dose reduction parameters in CCTA is given in table 4.

The options to reduce radiation dose are numerous and radiation dose is gradually declining. As stated before, radiation dose of CTA acquisition over time ranges from 2 to $30 \mathrm{mSv}$. [45] In more recent scanners with optimal, up-to-date scanning protocols, the radiation dose will generally be around 3 to $4 \mathrm{mSv}$ with a maximum of up to $7 \mathrm{mSv}$. [24, 25]

Radiation dose reduction

Scanner type (Multidetector, DSCT, etc.)

Tube current

Tube voltage

Triggering (Retrospective, prospective or ECG-gated tube current modulation)

High pitch helical scanning

Image reconstruction

scan range

Slice thickness

Pitch

Overlap

Acquisition time

Table 4. Factors in radiation dose reduction 


\section{Diagnostic and prognostic accuracy}

Scientific consensus documents have been published that address the appropriate use, diagnostic performance, prognostic value and interpretation of CCTA. [29, 48] Coronary artery calcium (CAC) is one of the parameters that can be assessed as part of a coronary CT acquisition protocol. As indicated, this involves a separate, non-contrast-enhanced CT scan prior to CCTA. The diagnostic and prognostic value of CAC was assessed in a systematic review by Sarwar et al. in 2009. [49] Only 146 of 25.903 asymptomatic individuals without CAC $(0.56 \%)$ experienced a cardiovascular event during mean follow-up of 51 months. In 7 studies assessing the prognostic value of CAC in a symptomatic population, $1.8 \%$ of the patients without CAC had a cardiovascular event during mean followup of 42 months. Furthermore, the combined 18 studies indicated that the presence of CAC had a sensitivity and negative predictive value of $98 \%$ and $93 \%$, respectively, for the detection of significant CAD on invasive coronary angiography. Prospective, population-based studies have shown that the calcium score is a very strong predictor of coronary events in asymptomatic individuals, with relative risks up to 10.

A systematic review and meta-analysis by Den Dekker et al. assessed the sensitivity and specificity of CCTA with 16-MDCT and newer CT scanner generations for significant stenosis at different degrees of coronary calcification. [50] 51 studies reported on the impact of calcium scoring on diagnostic performance of CCTA and were included the review. 27 out of 51 were suitable for the meta-analysis. Calcium scores were categorized as $0-100,101-400,401-1000$, and $>1000$. On a patient-basis, sensitivity of CCTA for significant stenosis was $95.8 \%, 95.6 \%, 97.6 \%$ and $99.0 \%$, respectively. Specificity of CCTA was $91.2 \%, 88.2 \%, 50.6 \%$ and $84.0 \%$, respectively, for 64-row MDCT and newer scanners. The 16-row MDCT generation performed significantly worse than the more recently introduced scanners. Specificity was lower in the group with a calcium score of 401-1000, mainly because of low numbers of patients. These results suggest that even in severely calcified coronary arteries, the sensitivity and specificity of CCTA for significant stenosis is high, in case of 64-row MDCT or newer scanners. A cut-off for calcium scoring for CCTA in newer CT systems no longer seems necessary.

A systematic review and meta-analysis from 2007 by Abdulla et al. evaluated the diagnostic accuracy of 64-multidetector CT compared with conventional invasive coronary angiography (ICA) for coronary stenosis. [51] Mean sensitivity and specificity of CCTA to detect and exclude significant stenosis on a patient basis were $98 \%$ and $91 \%$ respectively. Single center studies have shown that the negative predictive value (NPV) of CCTA for 64-row MDCT scanners is high (>95\%) and are clinically useful to rule out significant CAD. [15, 52, 53]

The diagnostic accuracy of ECG-gated 64-row MDCT in individuals without known CAD was assessed in a prospective multicenter trial named ACCURACY. [54] A total of 230 subjects underwent both CCTA and ICA. On a patient-based level the sensitivity, specificity, PPV and NPV to detect $>50 \%$ stenosis were $95 \%, 83 \%, 64 \%$ and $99 \%$ respectively. The test characteristics for $>70 \%$ stenosis were $94 \%, 83 \%, 48 \%$ and $99 \%$, respectively. 64-row MDCT 
was shown to have a high diagnostic accuracy in detecting coronary stenosis, both at $>50 \%$ and $>70 \%$. Even more importantly, the $99 \%$ NPV establishes CCTA as an alternative for ICA to rule out significant CAD.

Miller et al. (2008) also examined the accuracy of 64-row multidetector CT compared to conventional ICA. [55] In 291 patients with calcium score below 600, coronary segments with a diameter of $>1.5 \mathrm{~mm}$ were analyzed by CCTA and ICA. $56 \%$ of the patients had obstructive CAD. Patient-based diagnostic accuracy of CCTA for ruling out stenosis $>50 \%$ according to ICA revealed an area under the curve (AUC) of 0.93, with sensitivity, specificity, PPV, NPV of $85 \%, 90 \%, 91 \%$ and $83 \%$ respectively. The PPV and NPV in this study indicated that CCTA cannot replace ICA at that time.

Baumuller et al. compared CCTA with DSCT and 64-row MDCT in the diagnosis of significant coronary stenosis in 200 patients. [24] Of these patients, 100 underwent DSCT and 100 underwent MDCT. On a segment basis, sensitivity and specificity for DSCT were $96.4 \%$ and 97.4\% respectively. For 64-row MDCT the sensitivity and specificity were $92.4 \%$ and $95.3 \%$ respectively. The NPV for DSCT and 64-row MDCT was 99.5\% and 98.8\% respectively. DSCT showed significantly improved accuracy and specificity for the diagnosis of significant stenosis on a segment basis, but shows comparable diagnostic accuracy compared to 64-row MDCT on a per patient-analysis.

In a study by Leber et al., DSCT was performed in 88 patients. [20] Results showed an overall sensitivity and specificity on a segment base of $95 \%$ and $90 \%$ respectively. DSCT accurately ruled out coronary stenosis in patients with intermediate pretest likelihood for CAD, independent of the heart rate. Higher heart rates did not show significant decrease in diagnostic accuracy.

A non-comprehensive overview of studies regarding the diagnostic accuracy of CT (64-row and DSCT) for significant stenosis versus ICA is shown in table 5. It can be concluded from most studies that CCTA has a good sensitivity, specificity and excellent NPV. Thus, CCTA can be used to rule out or detect the presence of CAD in selected symptomatic populations suspected of CAD.

Another important aspect of CCTA is the possibility to predict coronary events in symptomatic patients. This may have consequences for clinical management. Hulten et al. performed a systematic review and meta-analysis on the prognostic value of CCTA. In 18 studies 9.592 patients were evaluated with a median follow-up time of 20 months. [63] Major adverse cardiac events (MACE) occurred at an absolute rate of $0.6 \%$ (myocardial infarction (MI) or death) in patients with negative scan results. Occurrence of MACE in the positive scan group was $8.2 \%$, with $\mathrm{MI}$ or death in $3.7 \%$. Adverse cardiovascular events among patients with a normal CCTA are very rare and comparable to the baseline risks among healthy patients. The negative likelihood ratio of CCTA with normal findings is comparable to reported values for stress myocardial perfusion scans and stress echocardiography. 


\begin{tabular}{llllll}
\hline Author & Sensitivity (\%) & Specificity (\%) & PPV (\%) & NPV (\%) & Number of patients \\
\hline Meijboom et al.[56] & 99 & 64 & 86 & 97 & 360 \\
\hline Miller et al.[55] & 85 & 90 & 91 & 83 & 291 \\
\hline Budoff et al.[54] & 95 & 83 & 64 & 99 & 230 \\
\hline Baumuller et al.[24] & 96.4 & 97.4 & 83.2 & 99.5 & 200 \\
\hline Tsiflikas et al.[57] & 94 & 79 & 88 & 90 & 170 \\
\hline Sun et al.[58] & 84.3 & 98.6 & 92.2 & 96.9 & 103 \\
\hline Ropers et al.[59] & 98 & 81 & 79 & 99 & 100 \\
\hline Brodoefel et al.[19] & 100 & 81.5 & 93.6 & 100 & 100 \\
\hline Weustink et al.[60] & 99 & 87 & 96 & 95 & 100 \\
\hline Leber et al.[20] & 95 & 90 & 74 & 99 & 88 \\
\hline Oncel et al.[53] & 100 & 100 & 100 & 99 & 80 \\
\hline Raff et al.[15] & 95 & 90 & 93 & 98 & 70 \\
\hline Ehara et al.[61] & 98 & 86 & 98 & 86 & 69 \\
\hline Mollet et al.[52] & 99 & 95 & 76 & 99 & 51 \\
\hline Achenbach et al.[62] & 100 & 82 & 72 & 100 & 50 \\
\hline Johnson et al.[22] & 100 & 89 & 89 & 100 & 35 \\
\hline
\end{tabular}

Table 5. Accuracy of DSCT and 64-row CT in the detection of coronary stenosis on a segment level in comparison to ICA.

\section{Indications}

Even though CCTA has only become a viable potential alternative for ICA for selected patients since the development of 64-MDCT, already a number of indications are supported by scientific societies, and the number of indications is rapidly increasing. In 2008, a report of a writing group deployed by the Working Group Nuclear Cardiology and Cardiac CT of the European Society of Cardiology (ESC) and the European Council of Nuclear Cardiology (ECNC) was written with the indications, applications, limitations and training requirements for CCTA analysis. [64] In 2010, the American College of Cardiology Foundation (ACCF) along with key- and subspecialty societies conducted an appropriate use review of common clinical scenarios where CCTA is frequently considered and applied. [48] For potential clinical applications, the advantages and disadvantages of CCTA must be weighed against ICA. The following section lists the potential clinical indications for the use of CTA. This list starts with the strongest indication and reports to less frequent and less strong indications, taking different opinions of aforementioned societies into account.

The strongest indication for CCTA is to rule out significant luminal stenosis in stable patients with suspected CAD, at low-intermediate pretest likelihood of disease. As stated above, literature convincingly demonstrates that CCTA has a high negative predictive value and allows to reliably rule out CAD. [48, 64-66] Here, the aim is to avoid ICA when CT shows the absence of clinically relevant CAD. Based on clinical and statistical considerations, CCTA will be most useful in patients with an intermediate likelihood of CAD. The false-positive rate may be too high, for patients with a very low pretest likelihood. In patients with high pretest likelihood, it is not likely that a negative CT result excludes significant CAD. 


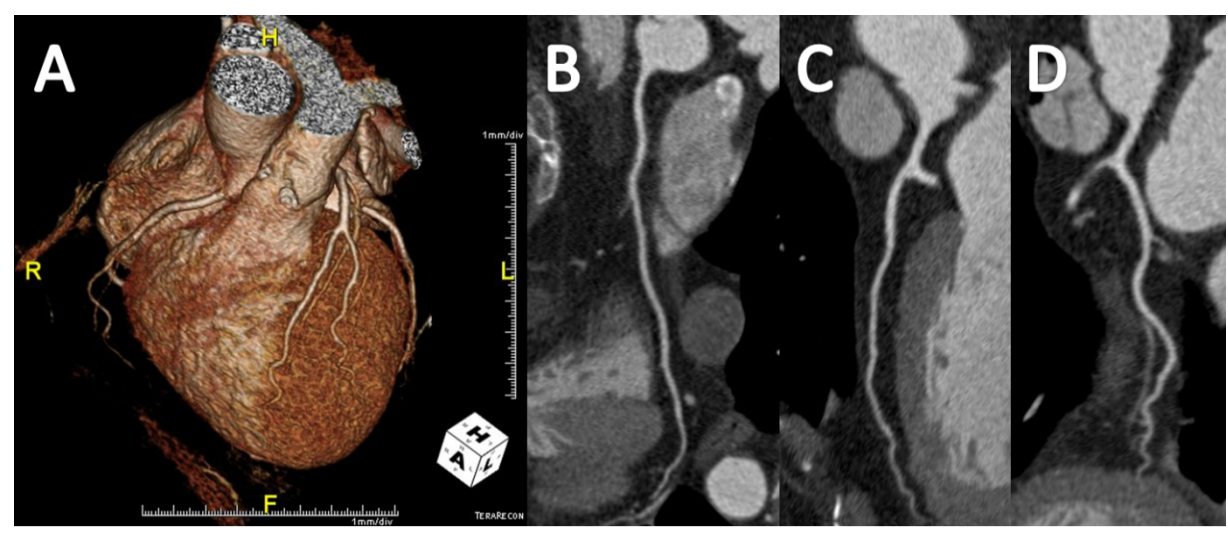

Figure 2. CCTA of a 61 year old women. A) 3D volume rendered reconstruction of the heart and the coronaries. The coronary arteries can be seen in the 3D reconstruction. B) Reconstruction of the right coronary artery (RCA) without CAD. C) Reconstruction of the left anterior descending artery of the same patient, also without stenosis. D) Circumflex (CX) reconstruction, not showing any disease.

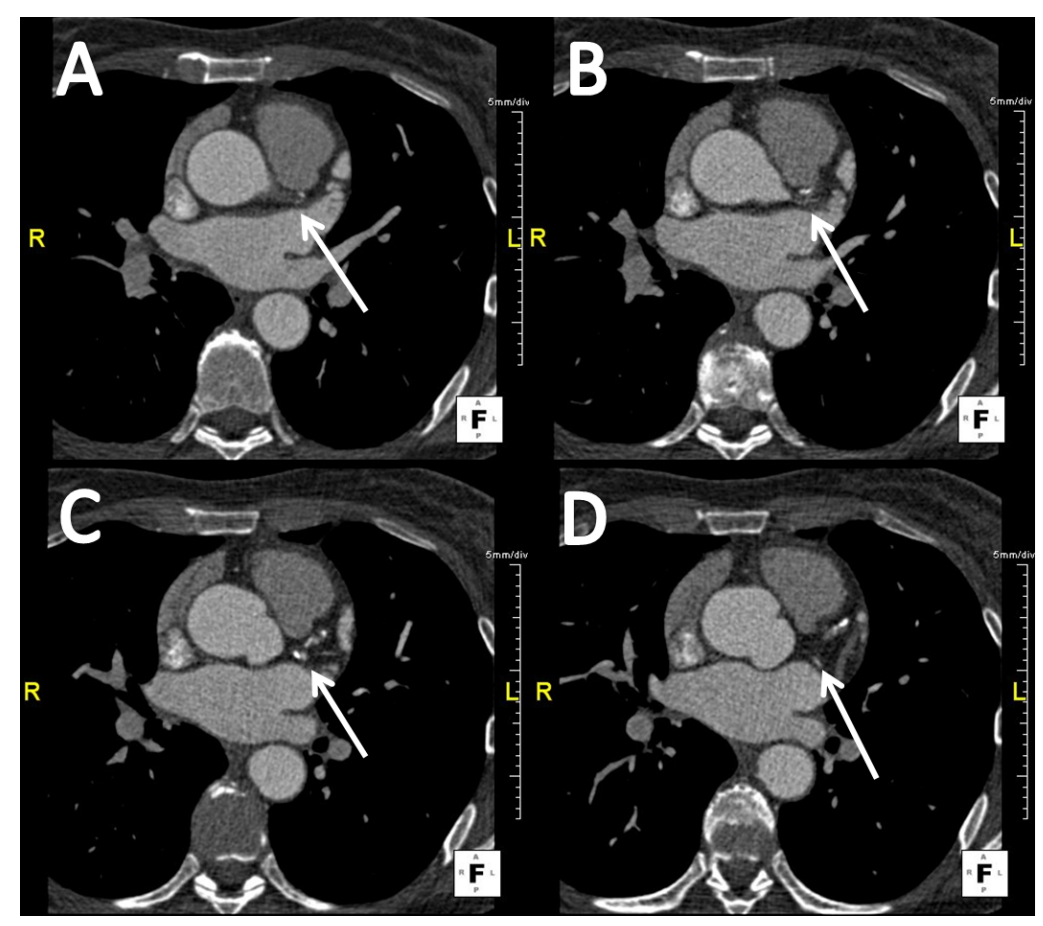

Figure 3. Overview of transverse CCTA images of a patient with an occlusion of the left main artery. In figs. A to C the arrow indicates the origin of the total occlusion. In image D the CX artery (retrograde filled) is pointed out. 
Another application of CCTA is to rule out CAD in acute chest pain. This concerns patients presenting to the emergency room with acute chest pain, without direct evidence of myocardial infarction based on e.g. electrocardiogram or myocardial enzymes. In these patients, further testing is often necessary in order to rule out significant CAD, or prolonged observation in the emergency room. Coronary CTA has been found useful in these patients to rapidly assess the coronary arteries for the presence of luminal stenosis. Recent studies have shown the efficiency of applying CCTA in the emergency room. $[67,68]$

CCTA can determine the complex course of anomalous coronary arteries. CCTA is the technique of choice for patient workup in known anomalous coronary vessels or vessels suspected to be anomalous because of the ease of data acquisition and the high resolution of the data set. CT has been qualified as an appropriate technique for evaluation of coronary anomalies. [48, 69-71] An example of CCTA analysis of coronary anomalies is shown in figure 4 .

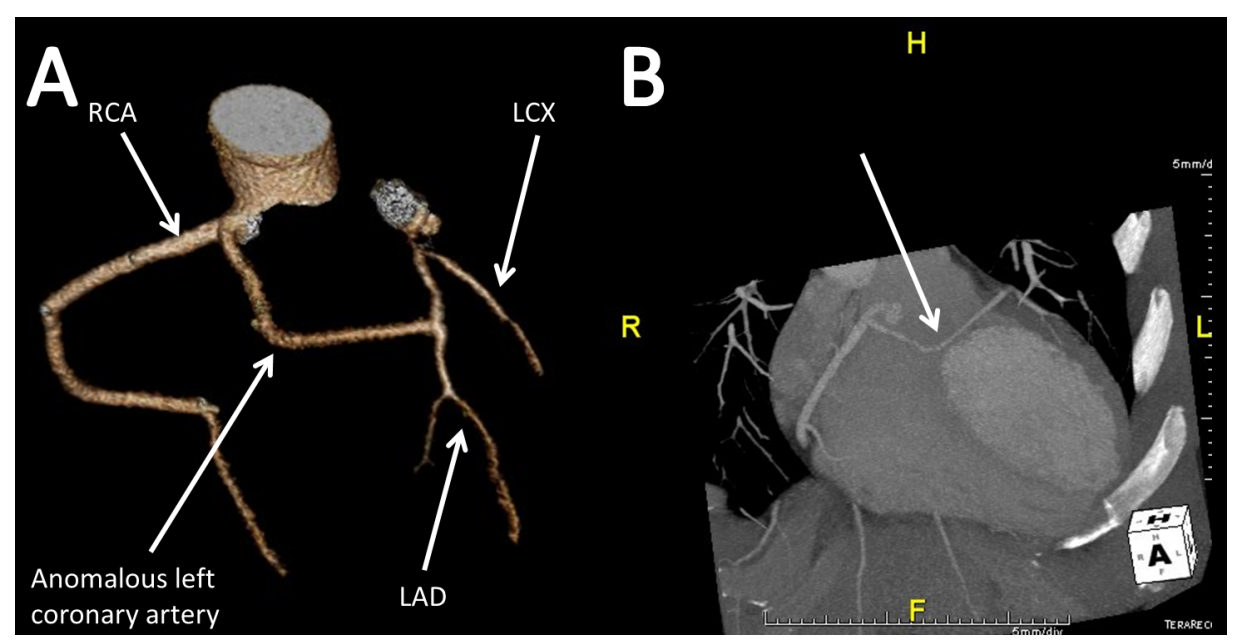

Figure 4. Patient with a coronary anomaly. A) The anomalous left coronary artery arises from the proximal RCA, through the septum into the LAD. B) Maximum intensity projection of the anomalous left coronary artery. 


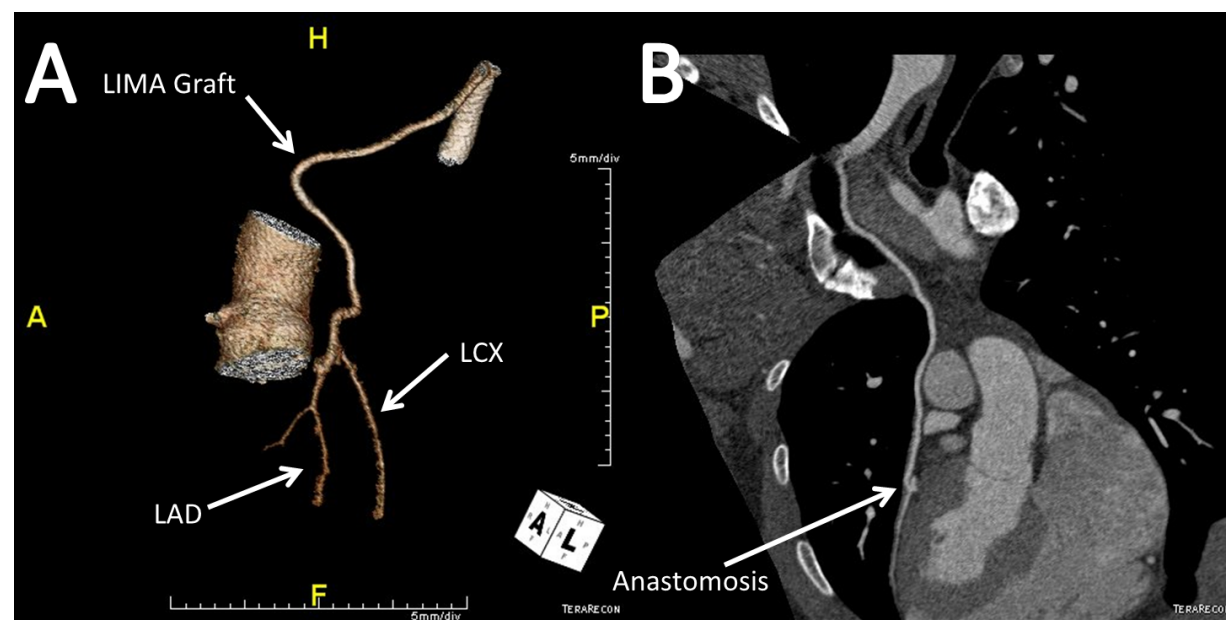

Figure 5. Evaluation after bypass-surgery, same patient as in figure 4. A) The distal left internal mammary artery (LI$M A)$ is anastomosed with the proximal LAD at the location of the former anomalous left coronary artery. B) The graft and distal LAD show good contrast filling.

Significant coronary artery disease, mainly left main disease, needs to be ruled out before non-coronary cardiac surgery. Cardiothoracic surgeons often request coronary angiography to rule out CAD in patients scheduled for cardiac surgery, for instance valve replacement. For this purpose, stress testing is not reliable enough, as ischemia could possibly be masked by the underlying pathology. CCTA may be a useful technique to analyze the coronary arteries, without having to perform an ICA. Meijboom et al. addressed the use of CCTA in the detection of CAD prior to aortic valve replacement. [72] The overall sensitivity and specificity of CCTA for detecting CAD was, respectively $100 \%$ and $82 \%$. So, it can be assumed that patients scheduled for cardiac surgery can be evaluated by CCTA for the detection of CAD, without subgroups such as arrhythmias and unstable patients.

It has been determined that CCTA has a high accuracy for the detection of bypass graft stenosis and occlusion. [73-77] Bypass grafts are arteries or veins from elsewhere in the patient's body, grafted to the coronary arteries to bypass coronary stenosis and improve blood supply to the myocardium, shown in figure 5. Especially venous grafts have a larger diameter and are less prone to motion, which is an advantage for image quality. Coronary artery calcifications and dimensions of native coronary arteries complicate assessment of native coronary arteries in patients with bypass grafts. Recent studies showed that the sensitivity and specificity is lower in bypass graft patients. [75] Therefore, in clinical cases in which only bypass graft evaluation is required, CCTA use may be beneficial. If the coronary arteries also require assessment, value of CCTA will be limited.

Recent statements agree on the value of coronary calcium scoring in asymptomatic individuals at intermediate risk of cardiovascular disease. In these patients, the calcium score has shown to improve risk stratification compared to cardiovascular risk factors. [36, 37] 
Multiple less frequent and less strongly supported applications for CCTA imaging are known. For instance, CTA can be used as an alternative when cardiac catheterization is impossible or carries too much risk. Percutaneous coronary intervention (PCI) planning could also be an indication for CCTA. CT can more reliably identify parameters influencing PCI outcome such as length and extent of the stenosis than ICA. [78] Assessment of coronary stent lumen is also a possibility with CCTA. The ability to assess the stents depends on many factors including stent type and diameter.

\section{CCTA Indications}

Detection of CAD in symptomatic patients with suspected CAD

Detection of CAD in asymptomatic individuals without known CAD

Detection of CAD in a newly diagnosed heart failure without known CAD

Rule out CAD before non-coronary cardiac surgery

Clarify unclear findings in other noninvasive imaging techniques

Assessment post CABG (Graft evaluation)

Assessment post $\mathrm{PCl}$ (Stent evaluation)

Evaluation of anomalies of coronary arterial and thoracic arteriovenous vessels

Evaluation of complex adult congenital heart disease

Evaluation of ventricular morphology and systolic function

Table 6. Table Indications for CCTA analysis

\section{Potential new application}

It is important to realize that the presence of a significant stenosis on CCTA does not equate with hemodynamically significant CAD. Not all stenoses result in reduced myocardial perfusion in stress, and not all patients with a positive ischemia test have coronary stenosis. Thus, whereas angiographic evaluation of coronary artery pathology (morphological information) is needed on the one hand, assessment of inducible ischemia (functional information) due to coronary narrowing is necessary on the other hand. The number of different examinations that a patient has to undergo may be considerably reduced by combining morphological and functional data acquisition in one technique. CT, PET/CT and SPECT/CT have the potential of providing both functional and morphological information. [79] CT perfusion imaging is still early in development. It has different imaging options such as dynamic perfusion CT and (static) dual energy CT. Dynamic perfusion CT acquires multiple images of the contrast buildup in the myocardium, which can be monitored. Myocardial segments perfused by a stenotic artery will have a slower and lower contrast upslope resulting in a hypodense area in the myocardium. In dual-energy imaging, the amount of iodine 
contrast in the myocardium can be derived based on images at different $\mathrm{KeV}$ energy levels, an indication of blood distribution in the myocardium.
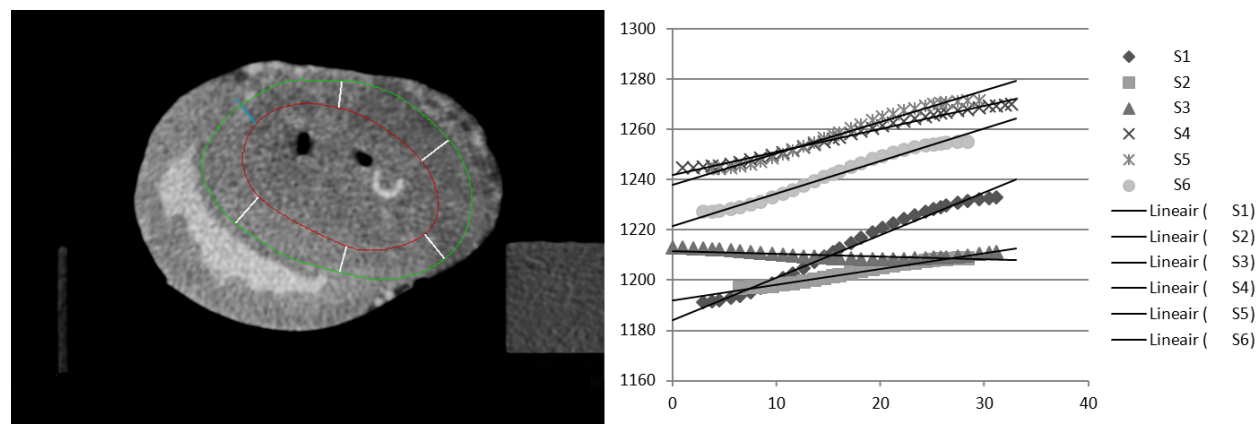

Figure 6. Dynamic CT perfusion analysis in a pig heart. The heart is divided into 6 segments ( 5 white lines and 1 blue line). Mean attenuation over time is monitored. B) Upslope of contrast enhancement in the 6 different segments in HU (not corrected for baseline -1024). Segments S2 and S3 have significantly lower upslope, corresponding with an applied stenosis in the CX artery.

\section{Conclusion}

Recent advances in modern CT technique have established CCTA as an accepted modality for coronary angiography in specific patients groups. One of the most important uses of CCTA is to exclude significant CAD in symptomatic patients at low-intermediate probability of significant stenosis. To yield most benefit from CCTA, patient selection remains important. Appropriate use will largely depend on patient characteristics, for instance pre-test likelihood of CAD. The advances in CT scanner technology have reduced the concerns about radiation dose, an important prior disadvantage. Exciting new imaging techniques in cardiac CTA could evolve in a comprehensive test for the assessment of CAD, making analysis of both anatomy and function possible in one modality.

\section{Author details}

G.J. Pelgrim ${ }^{1,2^{*}}$, M. Oudkerk ${ }^{2}$ and R. Vliegenthart ${ }^{1,2}$

*Address all correspondence to: g.j.pelgrim@umcg.nl

1 Department of Radiology, University of Groningen, University Medical Center Groningen, Groningen, Netherlands

2 Center for Medical Imaging - North East Netherlands, University of Groningen, University Medical Center Groningen, Groningen, Netherlands 


\section{References}

[1] Dodge JT, Jr., Brown BG, Bolson EL, Dodge HT. (1992) Lumen diameter of normal human coronary arteries. Influence of age, sex, anatomic variation, and left ventricular hypertrophy or dilation. Circulation; 86: 232-46.

[2] Harell GS, Guthaner DF, Breiman RS et al. (1977) Stop-action cardiac computed tomography. Radiology; 123: 515-7.

[3] Ritman EL, Kinsey JH, Robb RA et al. (1980) Three-dimensional imaging of heart, lungs, and circulation. Science; 210: 273-80.

[4] Keelan PC, Bielak LF, Ashai K et al. (2001) Long-term prognostic value of coronary calcification detected by electron-beam computed tomography in patients undergoing coronary angiography. Circulation; 104: 412-7.

[5] Guerci AD, Spadaro LA, Goodman KJ et al. (1998) Comparison of electron beam computed tomography scanning and conventional risk factor assessment for the prediction of angiographic coronary artery disease. Journal of the American College of Cardiology; 32: 673-9.

[6] Achenbach S, Giesler T, Ropers D et al. (2001) Detection of coronary artery stenoses by contrast-enhanced, retrospectively electrocardiographically-gated, multislice spiral computed tomography. Circulation; 103: 2535-8.

[7] Nieman K, Oudkerk M, Rensing BJ et al. (2001) Coronary angiography with multislice computed tomography. Lancet; 357: 599-603.

[8] Achenbach S. (2004) Detection of coronary stenoses by multidetector computed tomography: it's all about resolution. Journal of the American College of Cardiology; 43: 840-1.

[9] Flohr TG, Schoepf UJ, Kuettner A et al. (2003) Advances in cardiac imaging with 16section CT systems. Academic radiology; 10: 386-401.

[10] Kuettner A, Trabold T, Schroeder S et al. (2004) Noninvasive detection of coronary lesions using 16-detector multislice spiral computed tomography technology: initial clinical results. Journal of the American College of Cardiology; 44: 1230-7.

[11] Stein PD, Beemath A, Kayali F et al. (2006) Multidetector computed tomography for the diagnosis of coronary artery disease: a systematic review. The American journal of medicine; 119: 203-16.

[12] Herzog C, Britten M, Balzer JO et al. (2004) Multidetector-row cardiac CT: diagnostic value of calcium scoring and CT coronary angiography in patients with symptomatic, but atypical, chest pain. European radiology; 14: 169-77.

[13] Pugliese F, Mollet NR, Runza G et al. (2006) Diagnostic accuracy of non-invasive 64slice CT coronary angiography in patients with stable angina pectoris. European radiology; 16: 575-82. 
[14] Ong TK, Chin SP, Liew CK et al. (2006) Accuracy of 64-row multidetector computed tomography in detecting coronary artery disease in 134 symptomatic patients: influence of calcification. American heart journal; 151: 1323 e1-6.

[15] Raff GL, Gallagher MJ, O'Neill WW, Goldstein JA. (2005) Diagnostic accuracy of noninvasive coronary angiography using 64-slice spiral computed tomography. Journal of the American College of Cardiology; 46: 552-7.

[16] Herzog C, Nguyen SA, Savino G et al. (2007) Does two-segment image reconstruction at 64-section CT coronary angiography improve image quality and diagnostic accuracy? Radiology; 244: 121-9.

[17] Wintersperger BJ, Nikolaou K, von Ziegler F et al. (2006) Image quality, motion artifacts, and reconstruction timing of 64-slice coronary computed tomography angiography with 0.33-second rotation speed. Investigative radiology; 41: 436-42.

[18] Flohr TG, McCollough CH, Bruder H et al. (2006) First performance evaluation of a dual-source CT (DSCT) system. European radiology; 16: 256-68.

[19] Brodoefel H, Burgstahler C, Tsiflikas I et al. (2008) Dual-source CT: effect of heart rate, heart rate variability, and calcification on image quality and diagnostic accuracy. Radiology; 247: 346-55.

[20] Leber AW, Johnson T, Becker A et al. (2007) Diagnostic accuracy of dual-source multi-slice CT-coronary angiography in patients with an intermediate pretest likelihood for coronary artery disease. European heart journal; 28: 2354-60.

[21] Klepzig H. (2008) Diagnostic accuracy of dual-source multi-slice CT-coronary angiography in patients with an intermediate pretest likelihood for coronary artery disease. European heart journal; 29: 680.

[22] Johnson TR, Nikolaou K, Busch S et al. (2007) Diagnostic accuracy of dual-source computed tomography in the diagnosis of coronary artery disease. Investigative radiology; 42: 684-91.

[23] Nakashima Y, Okada M, Washida Y et al. (2011) Evaluation of image quality on a per-patient, per-vessel, and per-segment basis by noninvasive coronary angiography with 64-section computed tomography: dual-source versus single-source computed tomography. Japanese journal of radiology; 29: 316-23.

[24] Baumuller S, Leschka S, Desbiolles L et al. (2009) Dual-source versus 64-section CT coronary angiography at lower heart rates: comparison of accuracy and radiation dose. Radiology; 253: 56-64.

[25] Fink C, Krissak R, Henzler T et al. (2011) Radiation dose at coronary CT angiography: second-generation dual-source CT versus single-source 64-MDCT and first-generation dual-source CT. AJR American journal of roentgenology; 196: W550-7. 
[26] Mizuno N, Funabashi N, Imada M et al. (2007) Utility of 256-slice cone beam tomography for real four-dimensional volumetric analysis without electrocardiogram gated acquisition. International journal of cardiology; 120: 262-7.

[27] Dewey M, Zimmermann E, Deissenrieder F et al. (2009) Noninvasive coronary angiography by 320-row computed tomography with lower radiation exposure and maintained diagnostic accuracy: comparison of results with cardiac catheterization in a head-to-head pilot investigation. Circulation; 120: 867-75.

[28] Rybicki FJ, Otero HJ, Steigner ML et al. (2008) Initial evaluation of coronary images from 320-detector row computed tomography. The international journal of cardiovascular imaging; 24: 535-46.

[29] Abbara S, Arbab-Zadeh A, Callister TQ et al. (2009) SCCT guidelines for performance of coronary computed tomographic angiography: a report of the Society of Cardiovascular Computed Tomography Guidelines Committee. Journal of cardiovascular computed tomography; 3: 190-204.

[30] Pugliese F, Mollet NR, Hunink MG et al. (2008) Diagnostic performance of coronary $\mathrm{CT}$ angiography by using different generations of multisection scanners: single-center experience. Radiology; 246: 384-93.

[31] Hoffmann MH, Shi H, Manzke R et al. (2005) Noninvasive coronary angiography with 16-detector row CT: effect of heart rate. Radiology; 234: 86-97.

[32] Sun Z, Choo GH, Ng KH. (2012) Coronary CT angiography: current status and continuing challenges. The British journal of radiology; 85: 495-510.

[33] Agatston AS, Janowitz WR, Hildner FJ et al. (1990) Quantification of coronary artery calcium using ultrafast computed tomography. Journal of the American College of Cardiology; 15: 827-32.

[34] Budoff MJ, Shaw LJ, Liu ST et al. (2007) Long-term prognosis associated with coronary calcification: observations from a registry of 25,253 patients. Journal of the American College of Cardiology; 49: 1860-70.

[35] Greenland P, Bonow RO, Brundage BH et al. (2007) ACCF/AHA 2007 clinical expert consensus document on coronary artery calcium scoring by computed tomography in global cardiovascular risk assessment and in evaluation of patients with chest pain: a report of the American College of Cardiology Foundation Clinical Expert Consensus Task Force (ACCF/AHA Writing Committee to Update the 2000 Expert Consensus Document on Electron Beam Computed Tomography). Circulation; 115: 402-26.

[36] McClelland RL, Chung H, Detrano R et al. (2006) Distribution of coronary artery calcium by race, gender, and age: results from the Multi-Ethnic Study of Atherosclerosis (MESA). Circulation; 113: 30-7. 
[37] Elias-Smale SE, Proenca RV, Koller MT et al. (2010) Coronary calcium score improves classification of coronary heart disease risk in the elderly: the Rotterdam study. Journal of the American College of Cardiology; 56: 1407-14.

[38] Piers LH, Salachova F, Slart RH et al. (2008) The role of coronary artery calcification score in clinical practice. BMC cardiovascular disorders; 8: 38.

[39] Achenbach S, Boehmer K, Pflederer T et al. (2010) Influence of slice thickness and reconstruction kernel on the computed tomographic attenuation of coronary atherosclerotic plaque. Journal of cardiovascular computed tomography; 4: 110-5.

[40] Leipsic J, Labounty TM, Heilbron B et al. (2010) Adaptive statistical iterative reconstruction: assessment of image noise and image quality in coronary CT angiography. AJR American journal of roentgenology; 195: 649-54.

[41] Scheffel H, Stolzmann P, Schlett CL et al. (2012) Coronary artery plaques: cardiac CT with model-based and adaptive-statistical iterative reconstruction technique. European journal of radiology; 81: e363-9.

[42] Gutstein A, Wolak A, Lee C et al. (2008) Predicting success of prospective and retrospective gating with dual-source coronary computed tomography angiography: development of selection criteria and initial experience. Journal of cardiovascular computed tomography; 2: 81-90.

[43] Huang B, Li J, Law MW et al. (2010) Radiation dose and cancer risk in retrospectively and prospectively ECG-gated coronary angiography using 64-slice multidetector CT. The British journal of radiology; 83: 152-8.

[44] Hirai N, Horiguchi J, Fujioka C et al. (2008) Prospective versus retrospective ECGgated 64-detector coronary CT angiography: assessment of image quality, stenosis, and radiation dose. Radiology; 248: 424-30.

[45] Hausleiter J, Meyer T, Hermann F et al. (2009) Estimated radiation dose associated with cardiac CT angiography. JAMA : the journal of the American Medical Association; 301: 500-7.

[46] Hausleiter J, Meyer T, Hadamitzky M et al. (2006) Radiation dose estimates from cardiac multislice computed tomography in daily practice: impact of different scanning protocols on effective dose estimates. Circulation; 113: 1305-10.

[47] Siegel MJ, Schmidt B, Bradley D et al. (2004) Radiation dose and image quality in pediatric CT: effect of technical factors and phantom size and shape. Radiology; 233: 515-22.

[48] Taylor AJ, Cerqueira M, Hodgson JM et al. (2010) ACCF/SCCT/ACR/AHA/ASE/ ASNC/NASCI/SCAI/SCMR 2010 appropriate use criteria for cardiac computed tomography. A report of the American College of Cardiology Foundation Appropriate Use Criteria Task Force, the Society of Cardiovascular Computed Tomography, the American College of Radiology, the American Heart Association, the American Soci- 
ety of Echocardiography, the American Society of Nuclear Cardiology, the North American Society for Cardiovascular Imaging, the Society for Cardiovascular Angiography and Interventions, and the Society for Cardiovascular Magnetic Resonance. Journal of the American College of Cardiology; 56: 1864-94.

[49] Sarwar A, Shaw LJ, Shapiro MD et al. (2009) Diagnostic and prognostic value of absence of coronary artery calcification. JACC Cardiovascular imaging; 2: 675-88.

[50] den Dekker MA, de Smet K, de Bock GH et al. (2012) Diagnostic performance of coronary CT angiography for stenosis detection according to calcium score: systematic review and meta-analysis. European radiology.

[51] Abdulla J, Abildstrom SZ, Gotzsche O et al. (2007) 64-multislice detector computed tomography coronary angiography as potential alternative to conventional coronary angiography: a systematic review and meta-analysis. European heart journal; 28: 3042-50.

[52] Mollet NR, Cademartiri F, van Mieghem CA et al. (2005) High-resolution spiral computed tomography coronary angiography in patients referred for diagnostic conventional coronary angiography. Circulation; 112: 2318-23.

[53] Oncel D, Oncel G, Tastan A, Tamci B. (2007) Detection of significant coronary artery stenosis with 64-section MDCT angiography. European journal of radiology; 62: 394-405.

[54] Budoff MJ, Dowe D, Jollis JG et al. (2008) Diagnostic performance of 64-multidetector row coronary computed tomographic angiography for evaluation of coronary artery stenosis in individuals without known coronary artery disease: results from the prospective multicenter ACCURACY (Assessment by Coronary Computed Tomographic Angiography of Individuals Undergoing Invasive Coronary Angiography) trial. Journal of the American College of Cardiology; 52: 1724-32.

[55] Miller JM, Rochitte CE, Dewey M et al. (2008) Diagnostic performance of coronary angiography by 64-row CT. The New England journal of medicine; 359: 2324-36.

[56] Meijboom WB, Meijs MF, Schuijf JD et al. (2008) Diagnostic accuracy of 64-slice computed tomography coronary angiography: a prospective, multicenter, multivendor study. Journal of the American College of Cardiology; 52: 2135-44.

[57] Tsiflikas I, Brodoefel H, Reimann AJ et al. (2010) Coronary CT angiography with dual source computed tomography in 170 patients. European journal of radiology; 74: 161-5.

[58] Sun ML, Lu B, Wu RZ et al. (2011) Diagnostic accuracy of dual-source CT coronary angiography with prospective ECG-triggering on different heart rate patients. European radiology; 21: 1635-42.

[59] Ropers U, Ropers D, Pflederer T et al. (2007) Influence of heart rate on the diagnostic accuracy of dual-source computed tomography coronary angiography. Journal of the American College of Cardiology; 50: 2393-8. 
[60] Weustink AC, Meijboom WB, Mollet NR et al. (2007) Reliable high-speed coronary computed tomography in symptomatic patients. Journal of the American College of Cardiology; 50: 786-94.

[61] Ehara M, Surmely JF, Kawai M et al. (2006) Diagnostic accuracy of 64-slice computed tomography for detecting angiographically significant coronary artery stenosis in an unselected consecutive patient population: comparison with conventional invasive angiography. Circulation journal : official journal of the Japanese Circulation Society; 70: 564-71.

[62] Achenbach S, Goroll T, Seltmann M et al. (2011) Detection of coronary artery stenoses by low-dose, prospectively ECG-triggered, high-pitch spiral coronary CT angiography. JACC Cardiovascular imaging; 4: 328-37.

[63] Hulten EA, Carbonaro S, Petrillo SP et al. (2011) Prognostic value of cardiac computed tomography angiography: a systematic review and meta-analysis. Journal of the American College of Cardiology; 57: 1237-47.

[64] Schroeder S, Achenbach S, Bengel F et al. (2008) Cardiac computed tomography: indications, applications, limitations, and training requirements: report of a Writing Group deployed by the Working Group Nuclear Cardiology and Cardiac CT of the European Society of Cardiology and the European Council of Nuclear Cardiology. European heart journal; 29: 531-56.

[65] Hamon M, Biondi-Zoccai GG, Malagutti P et al. (2006) Diagnostic performance of multislice spiral computed tomography of coronary arteries as compared with conventional invasive coronary angiography: a meta-analysis. Journal of the American College of Cardiology; 48: 1896-910.

[66] Vanhoenacker PK, Heijenbrok-Kal MH, Van Heste R et al. (2007) Diagnostic performance of multidetector CT angiography for assessment of coronary artery disease: meta-analysis. Radiology; 244: 419-28.

[67] Meijboom WB, van Mieghem CA, Mollet NR et al. (2007) 64-slice computed tomography coronary angiography in patients with high, intermediate, or low pretest probability of significant coronary artery disease. Journal of the American College of Cardiology; 50: 1469-75.

[68] Hoffmann U, Truong QA, Schoenfeld DA et al. (2012) Coronary CT angiography versus standard evaluation in acute chest pain. The New England journal of medicine; 367: 299-308.

[69] Hollander JE, Litt HI, Chase M et al. (2007) Computed tomography coronary angiography for rapid disposition of low-risk emergency department patients with chest pain syndromes. Academic emergency medicine : official journal of the Society for Academic Emergency Medicine; 14: 112-6. 
[70] Deibler AR, Kuzo RS, Vohringer M et al. (2004) Imaging of congenital coronary anomalies with multislice computed tomography. Mayo Clinic proceedings Mayo Clinic; 79: 1017-23.

[71] Datta J, White CS, Gilkeson RC et al. (2005) Anomalous coronary arteries in adults: depiction at multi-detector row CT angiography. Radiology; 235: 812-8.

[72] Meijboom WB, Mollet NR, Van Mieghem CA et al. (2006) Pre-operative computed tomography coronary angiography to detect significant coronary artery disease in patients referred for cardiac valve surgery. Journal of the American College of Cardiology; 48: 1658-65.

[73] Nieman K, Pattynama PM, Rensing BJ et al. (2003) Evaluation of patients after coronary artery bypass surgery: CT angiographic assessment of grafts and coronary arteries. Radiology; 229: 749-56.

[74] Meyer TS, Martinoff S, Hadamitzky M et al. (2007) Improved noninvasive assessment of coronary artery bypass grafts with 64-slice computed tomographic angiography in an unselected patient population. Journal of the American College of Cardiology; 49: 946-50.

[75] Ropers D, Pohle FK, Kuettner A et al. (2006) Diagnostic accuracy of noninvasive coronary angiography in patients after bypass surgery using 64-slice spiral computed tomography with 330-ms gantry rotation. Circulation; 114: 2334-41; quiz

[76] Salm LP, Bax JJ, Jukema JW et al. (2005) Comprehensive assessment of patients after coronary artery bypass grafting by 16-detector-row computed tomography. American heart journal; 150: 775-81.

[77] Feuchtner GM, Schachner T, Bonatti J et al. (2007) Diagnostic performance of 64-slice computed tomography in evaluation of coronary artery bypass grafts. AJR American journal of roentgenology; 189: 574-80.

[78] Mollet NR, Hoye A, Lemos PA et al. (2005) Value of preprocedure multislice computed tomographic coronary angiography to predict the outcome of percutaneous recanalization of chronic total occlusions. The American journal of cardiology; 95: 240-3.

[79] Flohr TG, Klotz E, Allmendinger T et al. (2010) Pushing the envelope: new computed tomography techniques for cardiothoracic imaging. Journal of thoracic imaging; 25: 100-11. 